\title{
Damage phenomena of thin hard coatings submitted to repeated impacts: Influence of the substrate and film properties
}

\author{
S. Lamri ${ }^{\mathrm{a}, \mathrm{b}}$, C. Langlade ${ }^{\mathrm{a}, *}, \mathrm{G}$. Kermouche $^{\mathrm{c}, \mathrm{d}}$ \\ a IRTES-LERMPS, EA 7274, UTBM, 90010 Belfort Cedex, France \\ b ICD-LASMIS, UTT antenne de Nogent, Rue Lavoisier, 52800 Nogent, France \\ ${ }^{\mathrm{c}}$ LTDS Laboratory, UMR CNRS 5513, ENI St Etienne, France \\ ${ }^{\mathrm{d}}$ UMR CNRS 5146, Ecole des Mines de St-Etienne, Centre SMS, 158 Cours Fauriel, 42100 St-Etienne, France
}

\begin{abstract}
To evaluate the surface fatigue resistance of some thin nitride films obtained by Physical Vapour Deposition (PVD) techniques, repeated impact tests have been performed under controlled impact conditions. Short and long duration tests have revealed the occurrence of an original damage phenomenon likely linked to a mechanical blistering of the films. As these blisters appear to be the first damage step, their formation has to be understood in order to be avoided in industrial applications. In particular, the role of the mechanical properties of the substrate has to be clarified as thin protective coatings may be used on pieces prepared using various heat treatments. finite element method (FEM) analysis has been conducted in order to better understand the specific mechanical conditions in the substrate and at the film-substrate interface that could lead to such blistering phenomena. Correlations with the experimental results have been evidenced. From the modelling results the substrate properties have been shown to be of significant influence on the blister formation. However as they do not fully explain the origin of this phenomenon, the influence of the substrate microstructure has also been studied and the presence of vanadium carbides appears to be of major effect.
\end{abstract}

\section{Introduction}

Thin hard coatings are today well known solutions to improve surface performances and increase the component endurance life. Among them, nitride based films are one of the most popular coatings family due to their interesting mechanical properties. If various elaboration methods can lead to the realization of thin hard coatings on complex industrial parts, dry processes like Physical Vapour Deposition (PVD) or Chemical Vapour Deposition (CVD), are now widely used to coat mechanical parts submitted to severe tribological conditions. However, depending on the elaboration process and the particular deposition conditions, these films may exhibit very different performances. There is therefore a need for reliable characterisation methods, which could really give valuable information about the possible coatings in-service behaviour. Usual characterization methods like scratch or indentation tests are widely used but cannot satisfactorily describe the coatings long term performance [1,2]. To enable a better endurance life prediction, repeated impact testing strategies have been developed. Most of the research groups in domain are using high

\footnotetext{
* Corresponding author. Fax: + 33384583737.

E-mail address: cecile.langlade@utbm.fr (C. Langlade).
}

number of impacts of high energy to establish global S-N curves but without real insights in the damage origins [3,4]. In our case, attention has been focussed on the origin of the materials damage in order to be able to understand the initial phenomenon and its evolution law. Using this specific approach, not only endurance life curves (S-N) can be obtained [5,6] but also valuable information about the way to improve the coating performances. The present paper will focus on this last point.

During the first testing period, where no apparent damage can be evidenced, blisters appeared on some thin hard coatings tested under specific impact conditions. In the literature, blistering of films is mainly studied in relation with the surface preparation or elaboration process conditions [7-10]. Blisters of various geometrical shapes (round, telephone cord ...) may then be observed $[11,12]$. In the case of Thermal Barrier Coatings/oxides, uncontrolled elaboration parameters can lead to the creation of extremely severe compressive stresses in the growing coating that can induce spontaneous coating delamination and blistering. In extreme situations, complete spalling can be observed as soon as the deposition process stops [7,8]. Blistering phenomena may also be observed during oxidation experiments when the oxide films reach critical thickness values [13]. Mechanical models, related to the mechanical properties and thickness of the film, have been proposed to explain this blisters formation [11-14]. 
In [13], Strawbridge and Evans reviewed the different possible cases (thermal or mechanical origin, various blister shapes). In the case of a round (radius $R$ ) already fully de-laminated area, buckling is observed if the compressive residual stress in the film reaches a critical value $\sigma_{c}$ that can be determined by the following equation from [13]

$\sigma_{c}=\frac{122 E_{d}}{1-v_{d}^{2}}\left(\frac{e}{R}\right)^{2}$

where $\mathrm{e}$ is the thickness, $E_{d}$ the Young modulus, $v_{d}$ the Poisson coefficient of the film and $R$ the radius of the de-laminated area (insufficient adherence). From this equation, He. et al. [9] and Wang and Evans [15] proposed a buckling index $\Pi=\left(1-v^{2}\right) /(\sigma / E)(R / e)^{2}$ that characterizes the buckling risk for a delaminated coating. It is to be noted that this equation and the $\Pi$ index are not directly related to the reason of the film de-lamination that can be linked to an inappropriate elaboration process or to progressive crack propagation under mechanical conditions. Therefore, the strength of the coating/ substrate interface does not appear in Eq. (1) and is not used in numerical models [16-18]. However, this interfacial strength determines the blister stability that can be predicted using an adhesion index $\sum$ defined by Evans and co-workers in [19] as

$\sum=\sigma \sqrt{e(1-v) / E \Gamma_{i}}$

As all blister formed under our experimental impact conditions are round and stable, one can deduce from [19] that $\sum<1.85$ but no real estimation of the interfacial strength of our coating to be made. However, if the mechanical behaviour of a pre-delaminated film can be fully predicted [9-13] and local stress relief induced in films by buckling can be estimated [20], the specific conditions that lead to buckling under repeated impacts have to be clarified. In particular, the influence of the substrate hardness remains quite uncertain. If preliminary tests on M2 steels seem to indicate that a high hardness inhibits blister formation, results obtained on ductile C48 steel (AISI 1050) appear somehow contradictory. One unquestionable fact is that no blisters are observed on untested samples whatever the steel substrate nature and heat treatment. This suggests that the buckling conditions are induced by the impacts repetition.

The objective of the present work is then to better understand the conditions of the blister formation on various thin PVD hard nitride coatings and to evidence the influence of the substrate mechanical properties and/or microstructure on this blistering mechanism. Depending on the results, heat treatments or hardness, recommendations will be provided in order to avoid thin film blistering under impact conditions.

\section{Experimental}

\subsection{Impact tester}

The principle of the impact tester used in this study is sketched on Fig. 1 [5,6,21]. A rigid indenter, ended by a hemispherical tip electromagnetically is accelerated and pushed on the sample surface under normal incidence.

During this work, 100Cr6 (AISI 52100, which has the base composition of $1 \mathrm{C}-0.3 \mathrm{Si}-0.3 \mathrm{Mn}-1.5 \mathrm{Cr}(\mathrm{wt} \%)$ ) steel balls of $10 \mathrm{~mm}$ radius were used as the impacting tip leading to a total indenter mass of $165 \mathrm{~g}$. As a constant acceleration is generated by the electromagnets, the indenter velocity and kinetic energy just before the impact can be adjusted using the indenter initial position above the sample surface.

This incident velocity was also checked using a laser diode displacement sensor recording the tip position during each impact cycle [22]. Impact speeds ranging from 0.05 to $0.3 \mathrm{~m} / \mathrm{s}$ were used

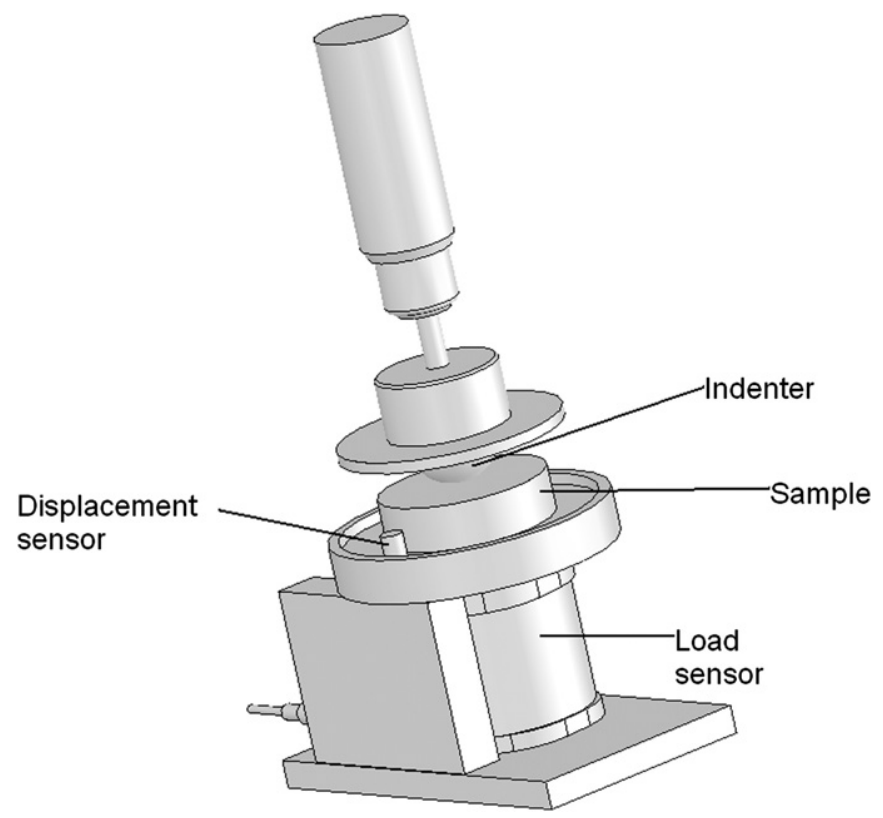

Fig. 1. Principle of the impact tester.

Table 1

Typical testing conditions.

\begin{tabular}{ll}
\hline Parameter & Range of values \\
\hline 100Cr6 ball radius & $10 \mathrm{~mm}$ \\
Kinetic energy (ball 165 g) & $0.2-8 \mathrm{~mJ}$ \\
Impact Force & $50-2500 \mathrm{~N}$ \\
Number of Impacts & $1-2 \times 10^{6}$ impacts \\
Impact Speed (m/s) & $0.05-3 \mathrm{~m} / \mathrm{s}$ \\
Impact Frequency & $10 \mathrm{~Hz}$ \\
\hline
\end{tabular}

during this study, leading to kinetic energies ranging from 0.2 to $8 \mathrm{~mJ}$. As the induced impact load depends not only on the kinetic energy of the indenter but also on the mechanical properties of the tested sample and the geometry of the indenter, it has to be determined for each testing condition. Due to the normal incidence and the limited penetration depth during each cycle (confirmed in the following by experimental and numerical results), only the normal component of the load (Fn) induced in the impacted material was considered and measured during each cycle using a piezoelectric transducer. Typical testing conditions are summarized in Table 1.

A set of reference testing conditions was chosen to be used for both modelling and experimental work in order to enable valuable comparisons. For these reference tests, the testing parameters were adjusted to ensure an incident kinetic energy of $0.8 \mathrm{~mJ}$ to enhance the influence of the mechanical properties of the substrate as well as the blistering phenomenon. Unless otherwise mentioned all results presented in the following were obtained under these reference conditions.

\subsection{Materials}

$\mathrm{CrN}$ thin coatings were elaborated at the LERMPS laboratory on annealed and treated (oil quenching $+1 \mathrm{~h} 30$ tempering at $550{ }^{\circ} \mathrm{C}$ ) M2 steel in order to achieve low $\left(230 \mathrm{Hv}_{50}\right)$ and high $\left(790 \mathrm{Hv}_{50}\right)$ substrate hardnesses. Each coating series were elaborated using the same experimental protocol (mirror polishing of the substrate, cleaning and deposition steps). The resulting film thickness was determined using a 3D optical profilometer. The Young modulus of the coatings was deduced from nanoindentation tests using a Nanoindenter XP2 
(Berkovich tip) from MTS. Tests were performed in the continuous stiffness measurement mode. Indentation results were analysed using the Oliver and Pharr determination procedure [23] and assuming a Poisson's coefficient of $v=0.25$ for the CrN coatings as commonly admitted in the literature $[24,25]$. Scratch tests were also performed using a Rockwell tip of $200 \mu \mathrm{m}$ and a loading speed of $100 \mathrm{~N} . \mathrm{min}^{-1}$ in order to estimate the critical load leading to the first crack formation. The main characteristics of the tested coatings are summarized in Table 2 .

\section{Experimental results and discussion}

Impact tests were performed on annealed and heat treated M2 coated steel to observe eventual differences in blistering phenomena. Both treated and untreated M2 steel samples were first coated with the same $\mathrm{CrN}$ film and tested under similar impact conditions.

\subsection{Untreated $M 2$ steel}

Fig. 2 presents the experimental observations performed on untreated M2 substrates coated with a $2.7 \mu \mathrm{m}$ thick $\mathrm{CrN}$ film.

Table 2

Coatings characteristics.

\begin{tabular}{lllll}
\hline Sample & $\begin{array}{l}\text { Film thickness } \\
(\mu \mathrm{m})\end{array}$ & $\begin{array}{l}\text { Young's modulus } \\
(\mathrm{GPa})\end{array}$ & $\begin{array}{l}\text { Hardness } \\
(\mathrm{GPa})\end{array}$ & $\begin{array}{l}\text { Scratch Lc } \\
(\mathrm{N})\end{array}$ \\
\hline $\begin{array}{l}\text { CrN/Annealed } \\
\text { M2 }\end{array}$ & 2.7 & $304 \pm 10$ & $25 \pm 3$ & 17.9 \\
CrN/Treated M2 & 2.8 & $300 \pm 10$ & $25 \pm 2$ & 19.5 \\
\hline
\end{tabular}

These micrographs clearly reveal that blisters can be formed even after a very low number of impacts (15 impacts for Fig. $2 a$ ) and even using impact energies as low as $0.6 \mathrm{~mJ}$. On increasing the impact energy (Fig. 2b), large spalling for the coatings can be observed. Moreover, the very first blisters are observed at the center of the impact scars, where the highest residual compressive stresses are expected from the modelling results.

\subsection{Treated M2 substrate}

Similar tests were performed on treated M2 samples also coated with a $2.8 \mu \mathrm{m}$ thick $\mathrm{CrN}$ film (identical elaboration process). The blistering of the $\mathrm{CrN}$ coating has been proved to be much more difficult to induce and to observe as illustrated in Fig. 3. Using impact energies as low as $0.6-0.8 \mathrm{~mJ}$ almost no blisters can be seen (Fig. 3a), even after several thousands of impacts (Fig. 3b). Impact tests at higher energy (up to $6.5 \mathrm{~mJ}$ ) do not significantly favour the blister formation (Fig. 3c). Spalling of the film can then be observed only for long duration tests. These impact tests, performed on heat-hardened steel seem to reveal the influence of the plastic deformation of the substrate in the blister creation.

\section{Finite element modelling (FEM)}

To better understand the buckling origin and in particular the specific mechanical conditions that can lead to this blister formation, a FEM analysis of the impact conditions was performed. The objectives of this modelling work were to observe what happens in the sample (film + substrate) during the repeated impacts that could induce the formation and/or growth of de-laminated areas leading to buckling. For this, a numerical compromise was to be found between an accurate stress and strain field description and a reasonable computation time to take into account several tens of

\section{a}

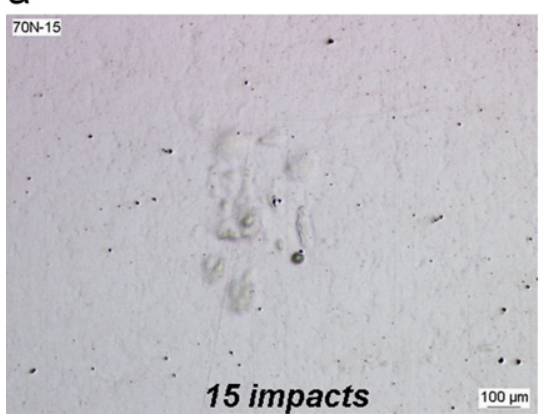

b

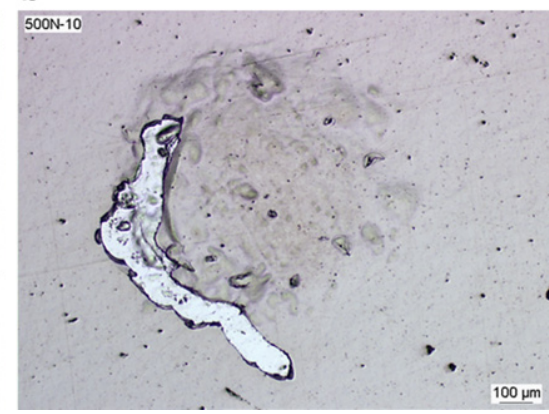

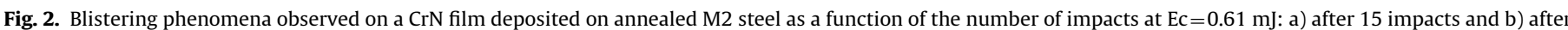
30 impacts.

a

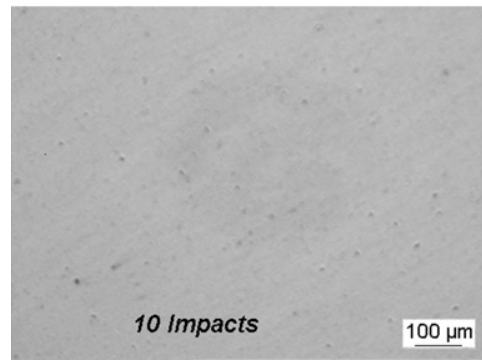

b

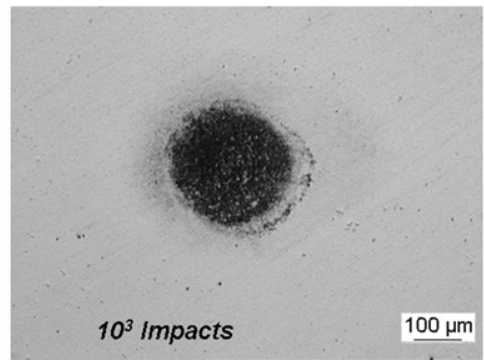

C

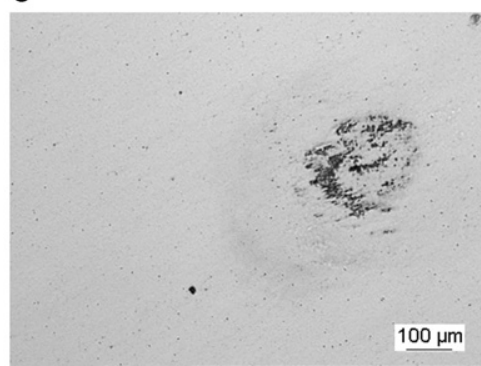

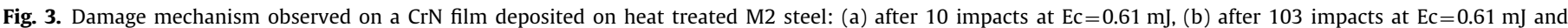
(c) after 10 impacts at $\mathrm{Ec}=3.26 \mathrm{~mJ}$. 
repeated impacts. The Abaqus explicit software version 6.8 [26] was chosen. Indeed, explicit finite element analysis is well adapted to fast nonlinear dynamic problems. Nevertheless, the time step in such analysis has to be small enough to maintain stability of the solution procedure [27]. In order to limit the Central Processing Unit time, the calculation was stopped when all the plastic deformation had occurred (end of the transient phase).

\subsection{Simulation step}

15 successive impact loadings were considered. Balls were assumed to be deformable spheres of $10 \mathrm{~mm}$ radius and an incident kinetic energy of $0.8 \mathrm{~mJ}$ was used for all calculations corresponding to an incident speed of $300 \mathrm{mms}^{-1}$ and a complete indenter weight (steel ball+sample holder) of $165 \mathrm{~g}$.

The thickness of the tested coated was experimentally determined (3D profilometry and SEM cross section examination) and varied from 2.7 to $3.2 \mu \mathrm{m}$. Therefore, a $3 \mu \mathrm{m}$ thick layer was considered on top of the steel samples. The length and width of the substrate were taken to be about 1000 times larger than the film thickness in order to eliminate any finite domain effect on numerical results. So a typical surface of $1.5 \times 2 \mathrm{~mm}^{2}$ was considered. Due to the specific geometry of the contacting parts and resulting contact, an axisymmetric model was used. An adapted mesh was preferred

a

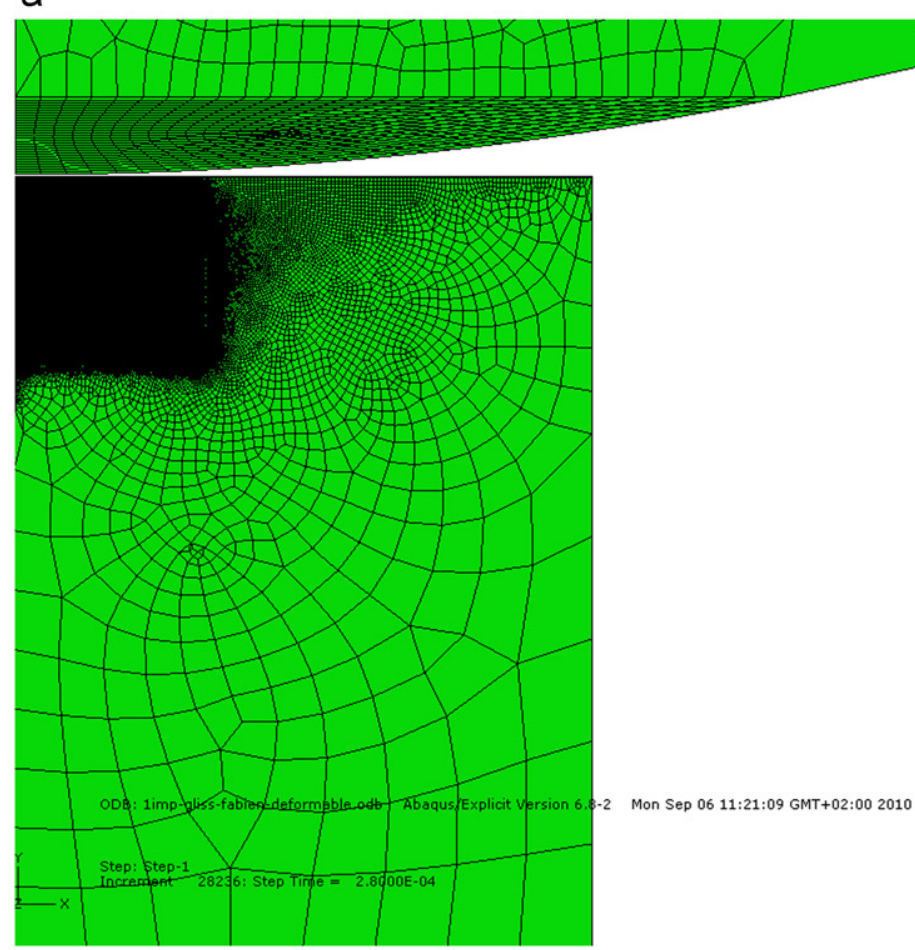

b
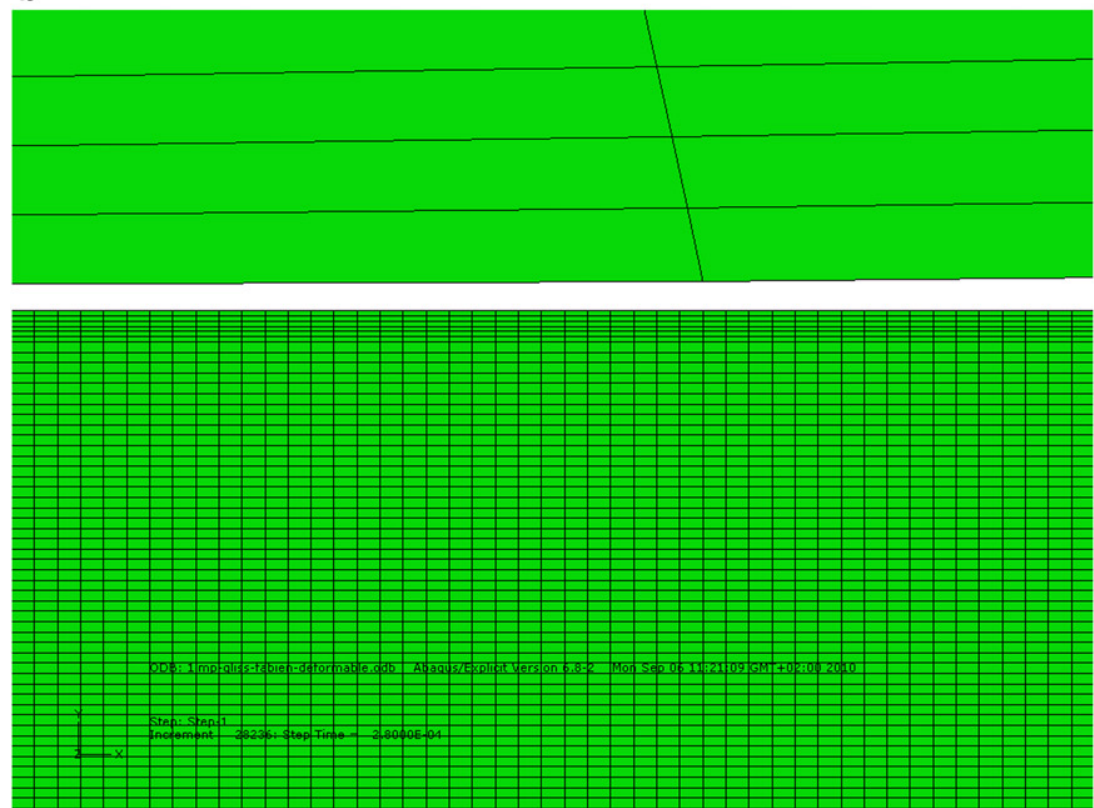

Fig. 4. FEM (Abaqus) mesh used for the study: (a) coarse mesh in the substrate and b) refined mesh in the film and near the interface. 


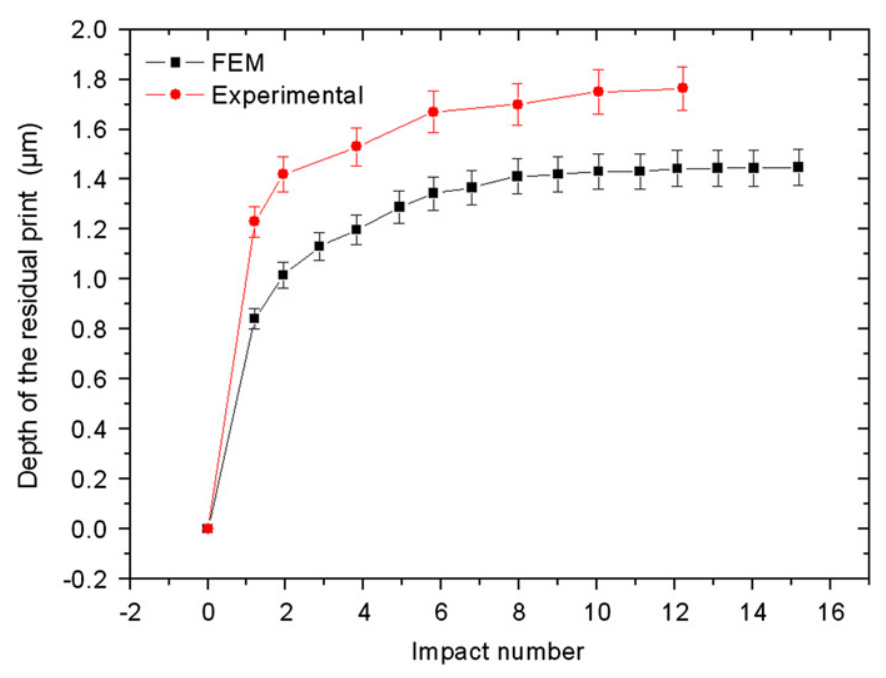

Fig. 5. Evolution of the residual scar depth for the first 15 impacts. using small elements in the film and at the interface vicinity and larger elements in the rest of the substrate as illustrated in Fig. 4. Boundary conditions were imposed in the radial (1) and axial (2) directions at the rear face of the sample. The symmetry axis was fixed in the (1) direction. Moreover, all rotations were forbidden for the impacting balls, in agreement with other FEM studies on similar ball on plane contacts [23-25]. Three types of materials (substrate, coating, and impacting balls) were implemented in the models. In particular, a Hollomon's law $\sigma=K \varepsilon^{n}$ was considered for the substrate mechanical behaviour along with a Young's modulus of $210 \mathrm{GPa}$ and Poisson's coefficient of 0.3 for the annealed M2 steel. The coefficients of Hollomon's law were determined using an inverse identification method [28] and set at $K=4500$ and $n=0.27$. This type of mechanical law was chosen as it enables accurate prediction of the evolution of normal load and scar depth that cannot be achieved using elastic- perfectly plastic laws. The mechanical behaviour of the $\mathrm{CrN}$ coatings was modelled using a perfect elastic law with a 300 GPa Young's Modulus obtained from nanoindentation results and a Poisson's coefficient of $v=0.25$ taken from the literature $[21,22]$.

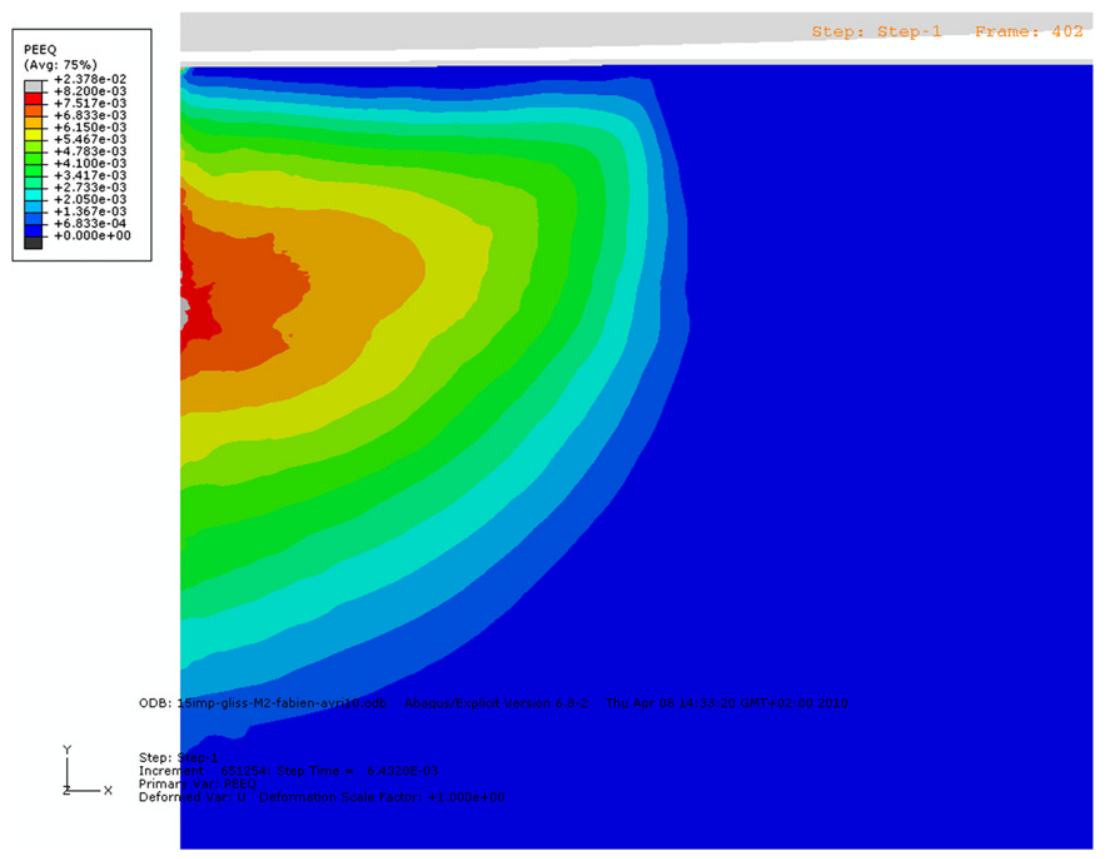

Fig. 6. Cumulated plastics strain distribution after 10 impacts.

a

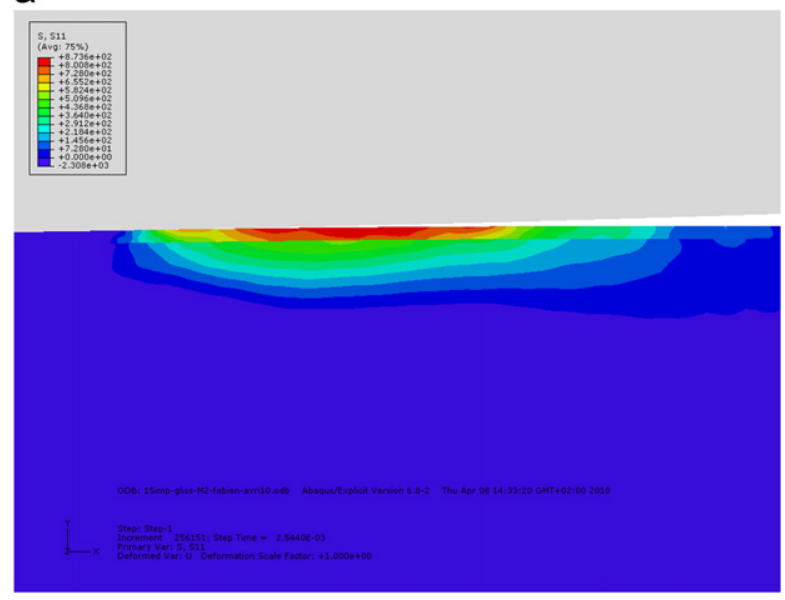

b

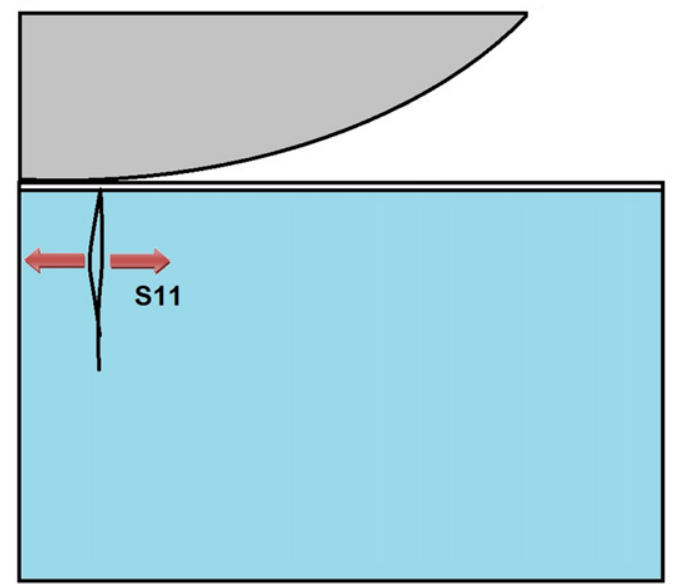

Fig. 7. (a) Distribution of the S11 stress field in the substrate during the backward motion of the indenter and (b) associated crack propagation model. 


\subsection{Transient impact regime}

Using these model and materials characteristics, the maximal residual depth observed during the first 12 impact cycles has been calculated and compared to experimental data (Fig. 5) obtained by 3D profilometry on impacted zones. It can be seen that, thanks to the chosen Hollomon's law, the residual depths of the impact scars are in good agreement with the results of numerical calculation.

The slight difference between experimental and numerical results can be related to slight impact velocity variation (typically less than 3\%) but also to wave reflections from boundaries in the finite element analysis. This may cause unexpected unloading of the indenter and thus leading to underpredicted values of indentation. During the very first impacts, plastic yielding occurs in the substrate. From the 8th impact, the material response appears to be stabilized and only elastic deformations are predicted. A steady-stable regime is then reached and the simulation predicts a residual depth in the 0.9-1.4 $\mu \mathrm{m}$ range. This phenomenon can be explained by a plastic accommodation due to the increase of the contact area per impact. This phenomenon is close to the process of cumulative plastic deformation under repeated rolling [29], whereby plastic deformation introduces residual stresses which make the elastic steady state virtually reached after a given number of cycles.

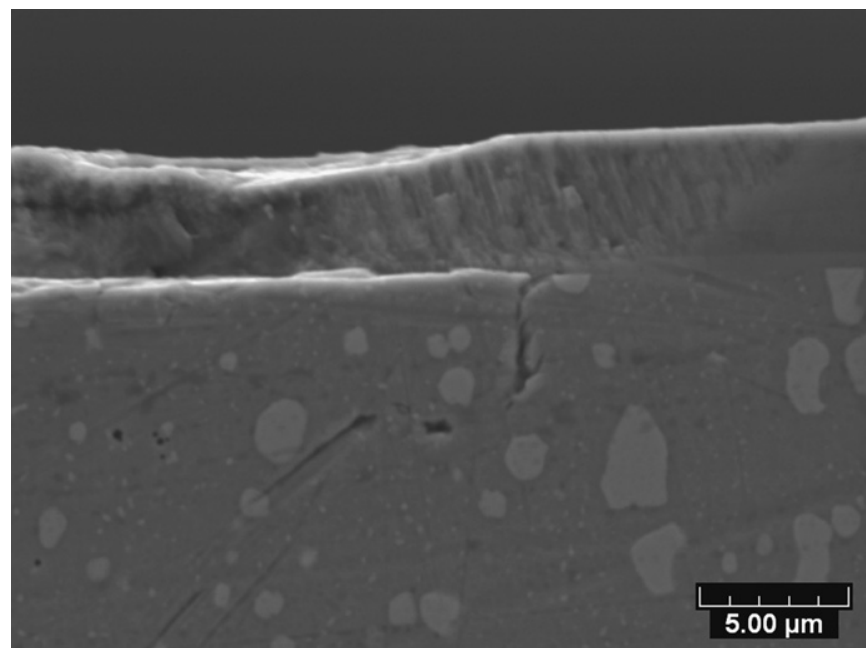

Fig. 8. Cross section micrography (SEM) of an impact scar on a $\mathrm{CrN}$ coated untreated M2 steel.

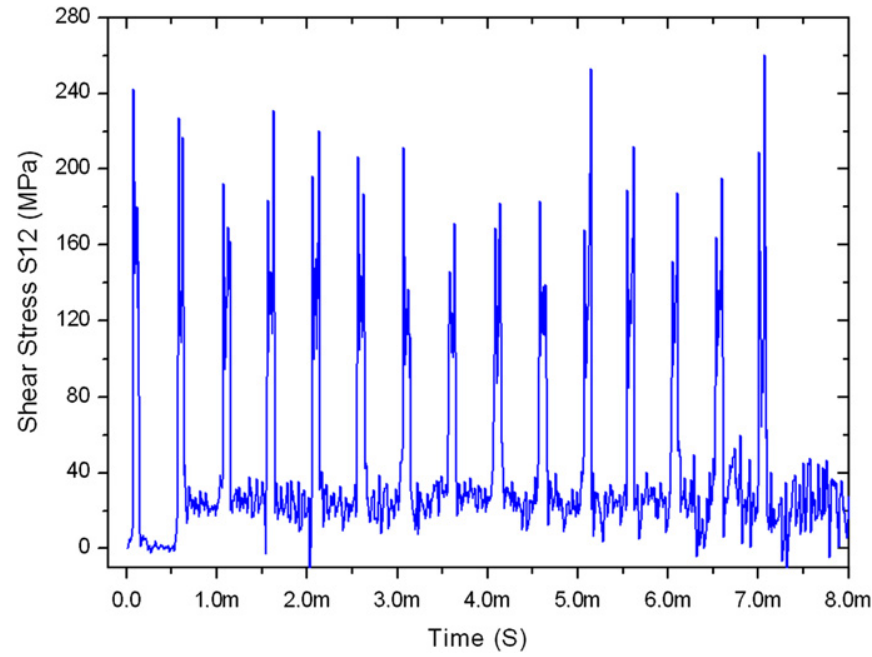

Fig. 10. Evolution of S12 stress field at the coating/substrate interface as a function of the number of impacts.

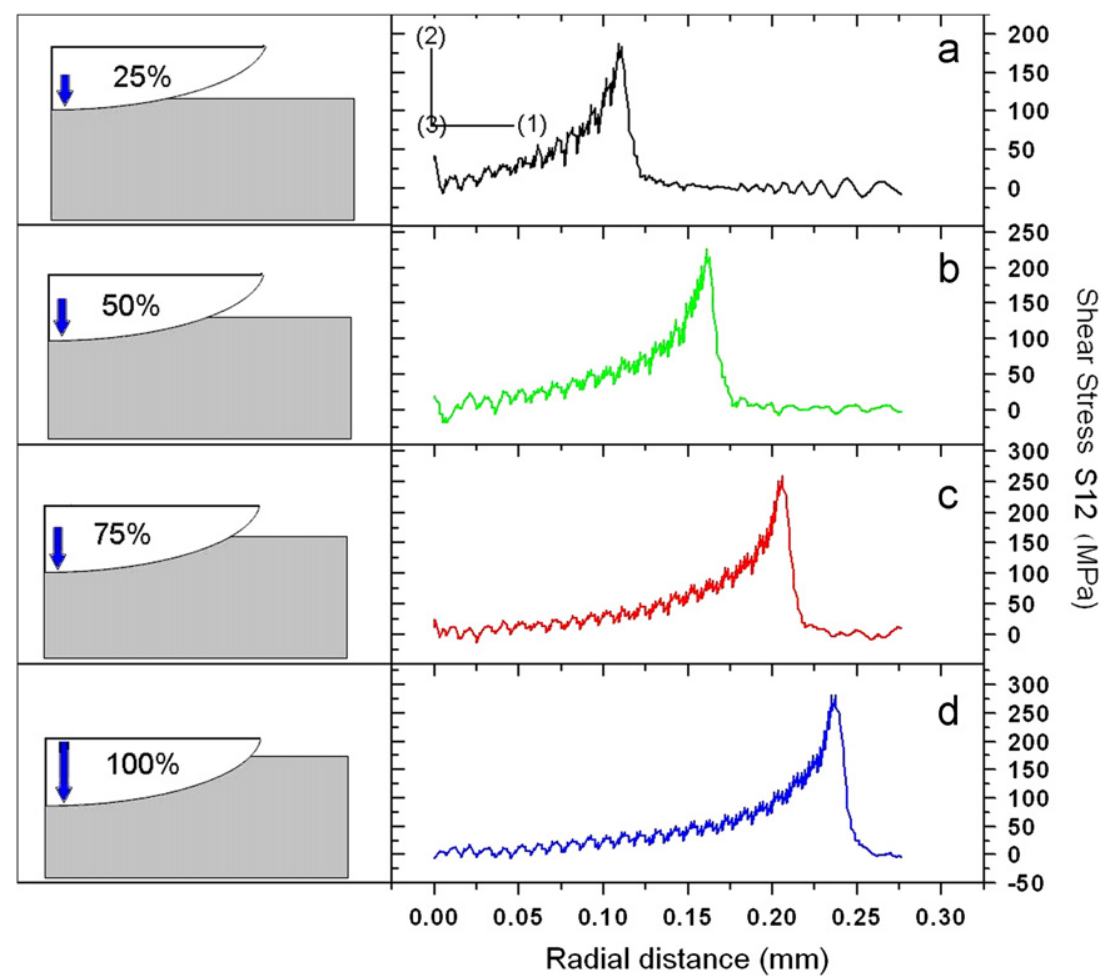

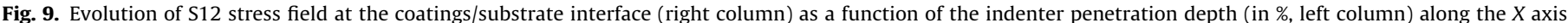
(a) for $25 \%$ penetration, (b) for $50 \%$ penetration, (c) for $75 \%$ penetration and (d) for $100 \%$ penetration, maximal depth. 


\subsection{Analysis of stress field during an impact}

\subsubsection{Substrate behaviour}

As no plastic deformations were expected in the hardened substrate (elastic behaviour), this first work focuses only on the case of untreated M2 substrates. Fig. 6 illustrates the distribution

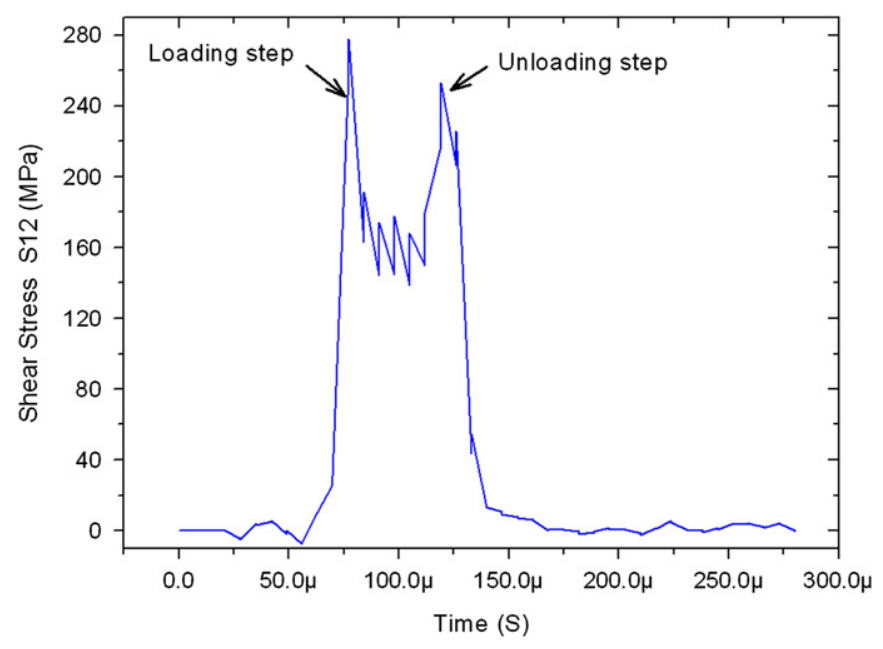

Fig. 11. Evolution of $S 12$ stress as a function of time during one impact.

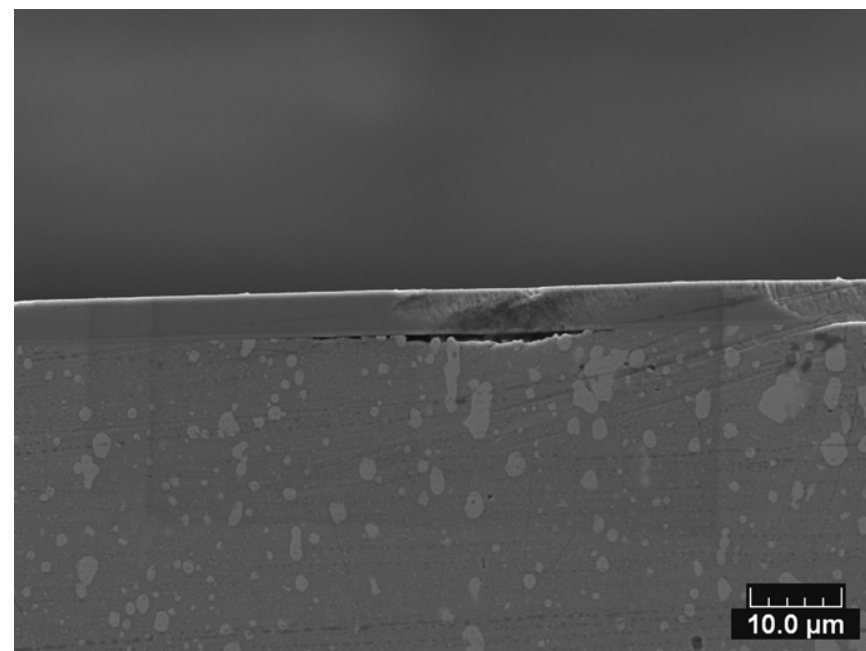

Fig. 12. SEM micrography of a delaminated zone on a tested $\mathrm{CrN}$ coated sample after 200 impacts under reference conditions. of plastic strain fields in the substrate. It clearly shows the presence of a plastic zone with very low distortion levels between $0.1 \%$ and $0.8 \%$ whereas an elastic zone can be observed just below the interface.

The computed S11 stress field for the untreated substrate (Hollomon's law) reveals the presence of a significant tensile stress in the film after the 10th impact with stress values ranging from 0 to $580 \mathrm{MPa}$ just below the interface. This residual tensile stress is a consequence of the plastic yielding in the substrate (Fig. 7a). These tensile stresses being repeated during each impact cycle can lead to the initiation of cracks that will propagate toward the interface as sketched in Fig. 7b.

To investigate the presence of possible cracks in the substrate that could be initiated and propagated by the repeated S11 tensile stress, cross section observations were made. Fig. 8 shows a SEM micrograph of a microcrack initiated in the substrate and propagated up to the interface detected on a tested sample after 2000 impacts at $0.8 \mathrm{~mJ}$. Such a crack can likely be related to the S11 repeated stress and, when reaching the interface, can initiate interfacial flaws.

\subsubsection{Interface behaviour}

FEM was also used to clarify the different stress fields which may develop at the substrate/coating interface during impact.

Fig. 9 presents the evolution of the $\mathrm{S} 12$ shear stress at the film/ substrate interface as a function of the penetration depth of the ball (in percent, $100 \%$ corresponding to the deepest penetration point) and the $x$ abscissa (in $\mathrm{mm}$ ) being the location of the observation point. During the penetration process (left column), the intensity of shear stress gradually increases (right column) and the point of application moves from the center of impact to the edge of the impact scar (Fig. 9a) up to $280 \mathrm{MPa}$ at $100 \%$ penetration of the ball (Fig. 9d). The existence of such a shear stress induced at the interface can have direct consequences on the mechanical behaviour of films.

A first analysis of these results seems to indicate that the presence and intensity of the S12 shear stress is directly linked to the plastic deformation undergone by the substrate. However, it can be noted that this shear stress distribution is obtained for the 10th impact (stabilized regime). The transient regime does not

Table 3

Composition of the used M2 steel.

\begin{tabular}{|c|c|c|c|c|c|}
\hline Composition & C & $\mathrm{Cr}$ & Mo & V & W \\
\hline$(w t \%)$ [31] & $0.78-0.88$ & $3.75-4.5$ & $4.5-5.5$ & $1.6-2.2$ & $5.5-6.75$ \\
\hline (wt\%) (EDS) & 1 & 4.11 & 5.26 & 2.17 & 6.49 \\
\hline
\end{tabular}

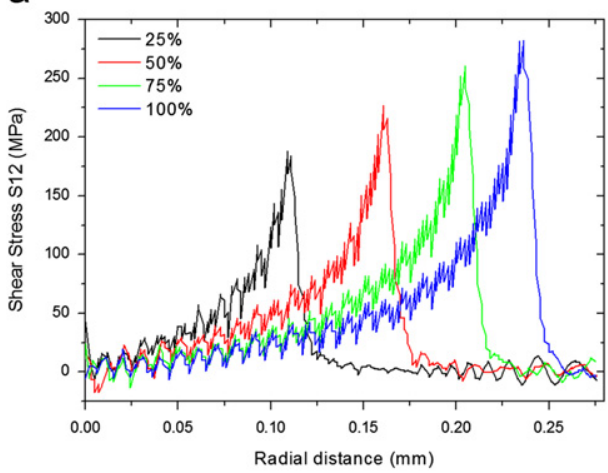

b

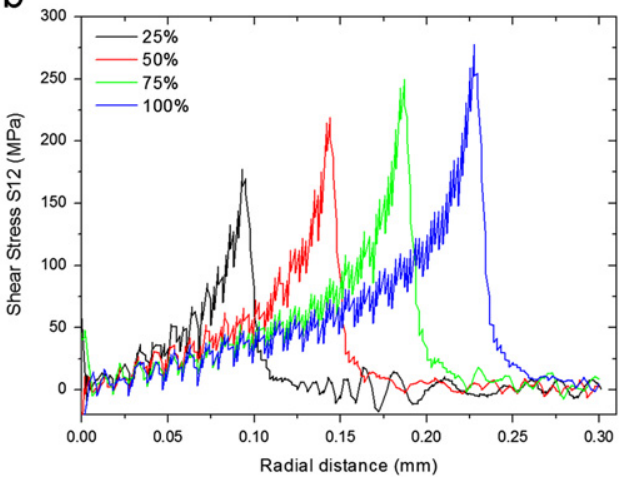

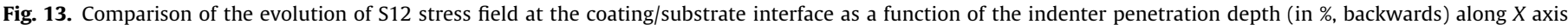
(a) Annealed M2 and (b) Heat treated M2. 
appear to influence the shear stress intensity. Fig. 10 presents the evolution of the S12 shear strain during the first 15 impacts and shows that the interface is subjected to shear stress during all impacts with a similar intensity $(220 \pm 20 \mathrm{MPa})$.

Analysis of the S12 shear stress at the interface during one impact (Fig. 11) shows that the interface is stressed twice per impact. A first stress peak is reached during the loading step and a second one occurs during the unloading phase, both having the same intensity $(260 \pm 10 \mathrm{MPa})$.

Two possible consequences of the presence of this tensile stress at the film/substrate interface can be imagined:

- the intensity of the induced shear stress exceeds the adherence strength of the coatings and generates the interface failure and film spalling;

- the intensity of the induced shear stress does not reach the adherence strength of the coatings but its repetition during each impact leads to the formation and propagation of interfacial cracks that will grow up to reach a critical size. By the combined effect of the residual compressive stresses present in the film (due to the elaboration process) and the induced tensile stress that acts during the impact unloading step, these critical interfacial defects will lead to the mechanical buckling of the film and the creation of blisters.

To confirm these numerical results, cross-sections observations of impacts scars were made. In Fig. 12, area of poor film/ substrate adhesion is observed whose growth can be mechanically activated and lead to the coating blistering.

\subsubsection{Influence of plastic deformation of substrate on shear stress}

When a Hollomon's law is considered for the substrate mechanical behaviour, S12 shear stresses are created throughout the interface during the loading and unloading steps. We can assume that these shear stresses will favor the observed film blistering. To validate this result, the case of a hard substrate with no plastic deformation under impact was also considered.

A perfect elastic behavior law was used for the hardened substrate. Fig. 13 presents the evolution of the S12 shear stress at

a

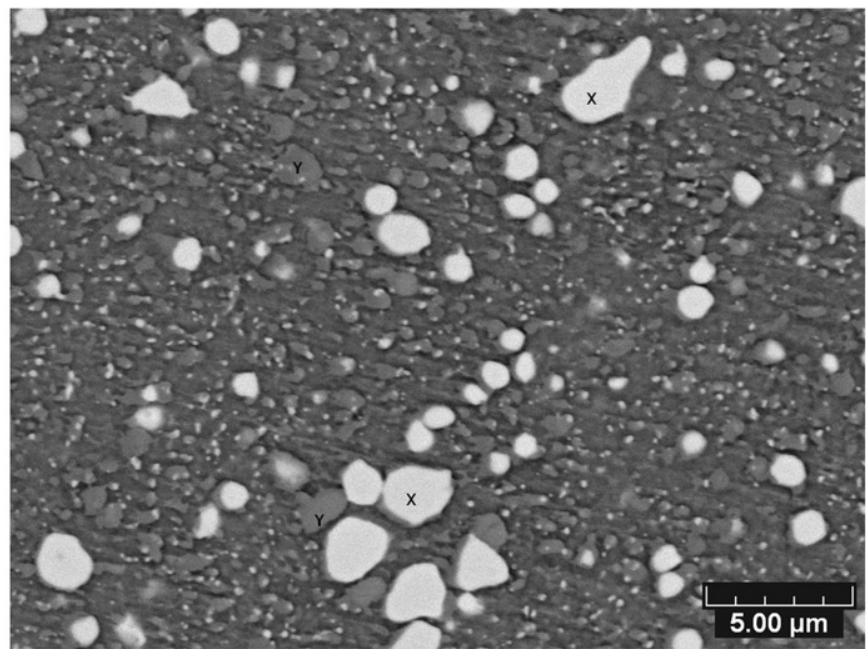

b

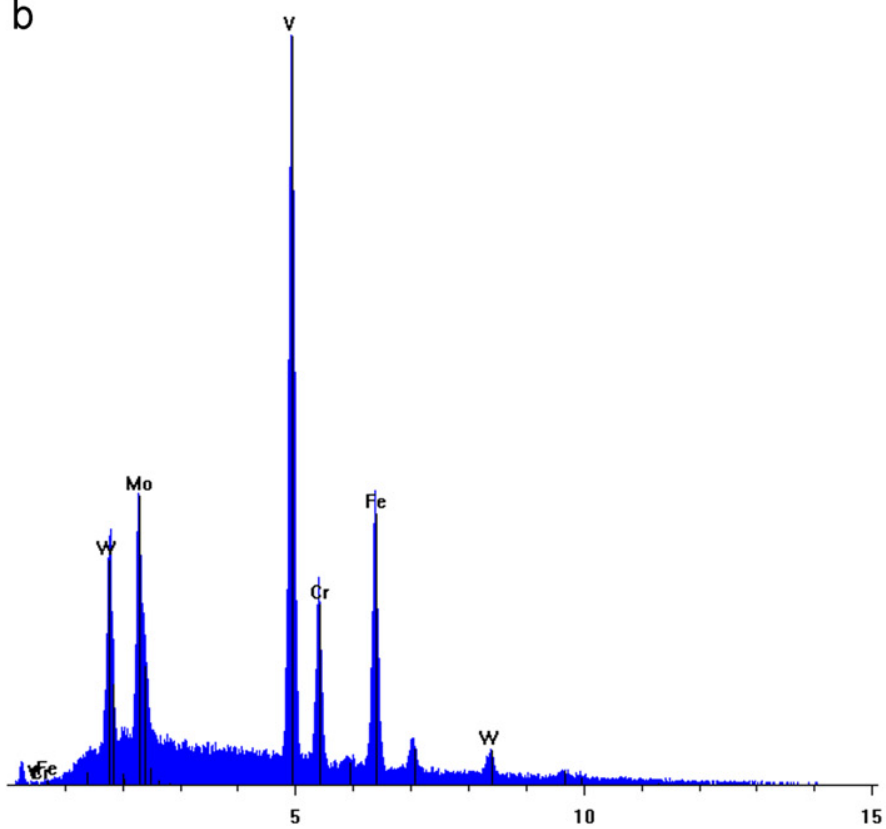

C

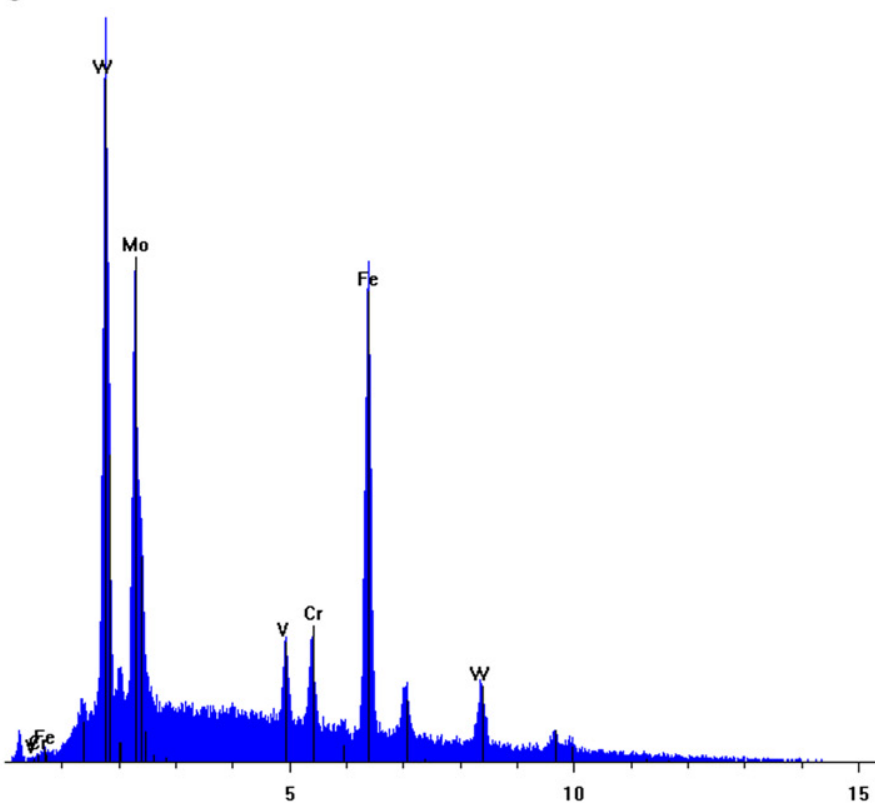

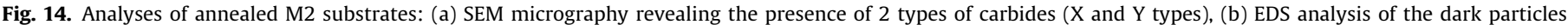
(Y type) and (c) EDS analysis of the white particles (X type). 
the film/substrate interface as a function of the penetration depth of the ball and the $x$ abscissa (in $\mathrm{mm}$ ) of the location of the observation point for annealed substrates (Hollomon's law, Fig. 13a), and treated substrates (elastic behaviour, Fig. 13b). It is found that the evolutions of the S12 shear stress along the interface are of the same order of magnitude for the two types of substrates. The shear stress intensity increases with the penetration of the indenter, ranging from $170 \mathrm{MPa}$ to $280 \mathrm{MPa}$ when the penetration increases from $25 \%$ to $100 \%$, respectively and its application point also moves along the interface. This means that the shearing of the interface does not depend on the mechanical behavior of the substrate. One can conclude that the plastic deformation of the substrate is not the main cause of the formation of blisters after impact.

\section{Influence of substrate structure}

Since the plasticity of the substrate does not seem to be at the origin of the observed differences in the blister occurrence, the influence of the substrate microstructure (annealed or treated M2) on the creation of defects and de-laminated zones has been studied.

In PVD hard coatings, the growth defect phenomena are very well known and have been studied by various authors [30,31]. They have found that the main factors which influence defect density are the cleanness of the chamber, the ion cleaning step, the sputtering power and shielding of the substrates. Any imperfections formed on substrate surface during pretreatment or growth defects are drawbacks for future applications as they can cause local loss of adhesion.

As flaws likely form on microstructure heterogeneities, a finer material analysis was conducted by scanning electron microscopy (SEM) coupled with energy dispersive spectroscopy (EDS). The nominal and analysed compositions of the M2 steel are given in Table 3.

M2 steels are characterized by an average carbon content around $0.8 \%_{w t}$, combined with alloying elements such as molybdenum, vanadium and tungsten which give good wear resistance through the formation of very hard carbides [32].

Fig. 14a presents the microstructure of annealed substrates obtained by scanning electron microscopy (SEM) showing the existence of two populations of carbides (X and Y areas in Fig. 14). EDS analyses reveal that they represent $36 \%$ of the surface, of which $9 \%$ are carbides of molybdenum and tungsten (Fig. 14b) and $27 \%$ are vanadium carbides (Fig. 14c. Cekada et. al. [33] have shown that the sputtering rate of molybdenum-tungsten carbide in HSS tool steel is higher than that of the matrix, while the sputtering rate of vanadium carbide is lower. These different sputtering rates during the cleaning pretreatment create defects with different depths in the substrate. This differential effect could be responsible for the presence of area of poor adhesion between the $\mathrm{CrN}$ film and the substrate. The high proportion of carbides in the M2 steel substrates seems to contribute to the creation of flaws, since there are carbides of molybdenum, tungsten and vanadium at the interface.

The microstructure of heat treated M2 substrates is shown in Fig. 15a. In addition to the martensitic structure, there is a significant decrease in the amount of carbides in the matrix. Only carbides of molybdenum and tungsten (Fig. 15b) appear in the matrix with a ratio of $4 \%$.

The lower carbides content in the hardened steel matrix can be responsible for the better adhesion of the $\mathrm{CrN}$ films and especially the reduction of defects at the interface enhancing the coating adhesion.

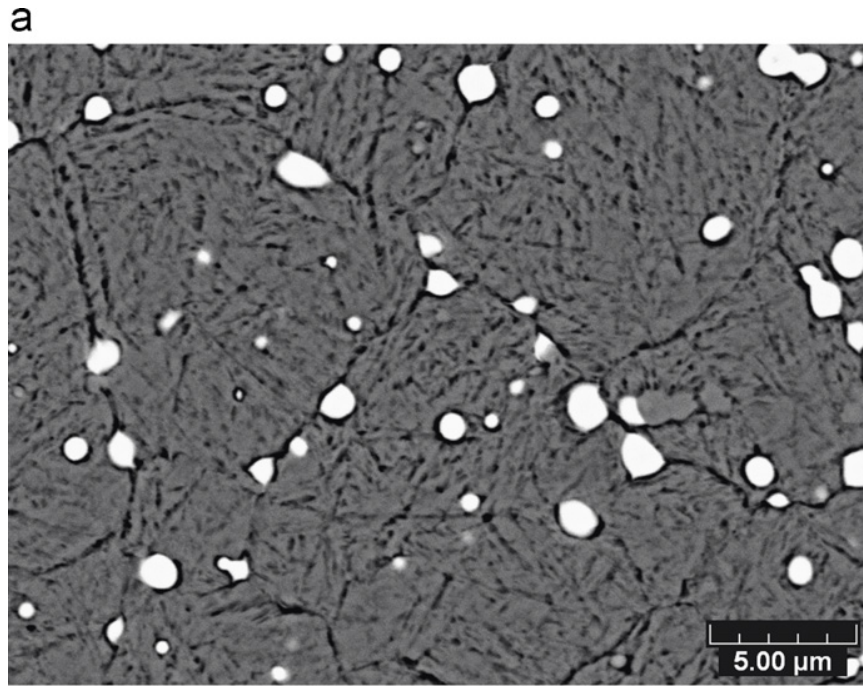

b

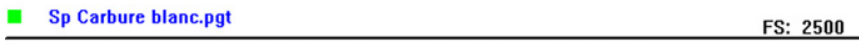

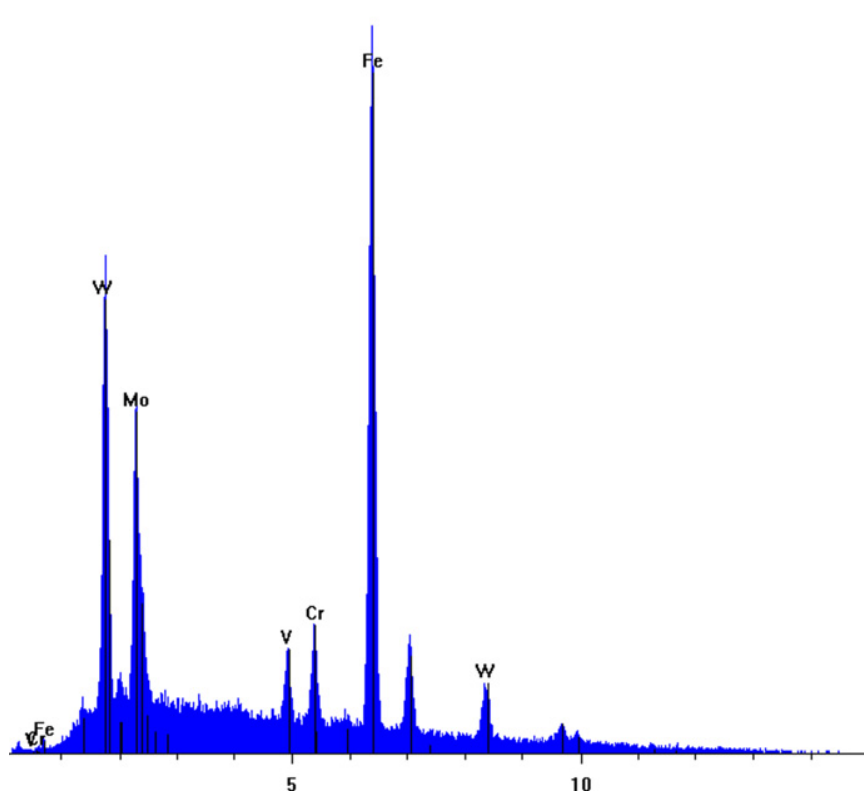

Fig. 15. Analyses of heat treated M2 substrates: (a) SEM micrography and (b) EDS analysis of the particles.

\section{Conclusions}

In this paper, it has been shown that the substrate properties play a major role in the blistering mechanism of thin CrN PVD films under repeated impacts. Both experimental and numerical works confirm the influence of the substrate mechanical properties. However, the material microstructure and in particular the presence of various types of carbides appear to be of major effect. The present work especially enables to establish that:

- If the impact energy is high enough to induce plastic deformations of the substrate, a radial tensile stress is observed in the substrate just below the interface. This tensile stress being repeated during each impact cycle can lead to the initiation of cracks that will propagate towards the interface. The presence of high carbides content (brittle particles) in the annealed M2 substrates can enhance this crack initiation process as 
confirmed by cross section observations on impacts scars that reveal the presence of such microcracks in the substrate.

- Whatever the substrate properties are, S12 shear stress fields of similar amplitude range are created at the coating/substrate interface. If these shear stresses can clearly favour the interfacial flaws creation and growth leading to the blister formation, their almost identical values cannot explain the difference in blistering occurrence for the two different M2 substrates. As hardened M2 substrates do not lead to any blister formation even when subjected to similar interfacial shear stress field during impacts, the reason of the easy blistering effect on annealed substrates is not to be found in these $\mathrm{S} 12$ shear stresses.

- The difference in microstructure between the two sets of samples (annealed and heat treated), especially the nature and content ratio of the carbide particles, can lead to the interfacial flaws formation. These defects, submitted to the repeated S12 shear stress, can then grow until reaching the critical blistering size.

\section{Acknowledgements}

The authors wish to thank the PMA (Pays de Montbéliard Agglomération) and UTBM for their financial support. The acquisition software of the impact tester has been financially supported by an OSEO project (No. 082009I) and was realized by P. Nöel (UTBM). This study is part of a Ph.D. work granted by the French Education Ministry. The UTBM-ENISE cooperation benefits from the financial support of the CNRS (French National Research Council) through an ITC program of the Plasma Froids network.

\section{References}

[1] K. Fujita, A. Yoshida, Wear 51 (1978) 365-374.

[2] S. Fouvry, V. Fridrici, C. Langlade, P. Kapsa, L. Vincent, Tribol. Int. 39 (2006) 1005-1015.
[3] O. Knotek, B. Bosserhoff, A. Schrey, T. Leyendecker, O. Lemmer, S. Esser, Surf. Coat. Technol. 54-55 (1992) 102-107.

[4] K.-D. Bouzakis, N. Vidakis, D. Kallinikidis, T. Leyendecker, G. Erkens, R. Wenke, H.-G. Fuss, Surf. Coat. Technol. 108-109 (1998) 526-534.

[5] F. Ledrappier, C. Langlade, A.-B. Vannes, Y. Gachon, Plasma Process. Polym. 4 (2007) S835-S839.

[6] F. Ledrappier, C. Langlade, Y. Gachon, B. Vannes, Surf. Coat. Technol. 202 (2007) 1789-1796.

[7] B.L. French, J.C. Bilello, Thin Solid Films 446 (2004) 91-98.

[8] X.F. Yang, D.E. Tallman, G.P. Bierwagen, S.G. Croll, S. Rohlik, Polym. Degrad. Stab. 77 (2002) 103-109.

[9] M.Y. He, A.G. Evans, J.W. Hutchinson, Mater. Sci. Eng. A 245 (1998) 168-181.

[10] M.-W. Moon, J.-W. Chung, K.-R. Lee, K.H. Oh, R. Wang, A.G. Evans, Acta Mater 50 (2002) 1219-1227.

[11] C. Coupeau, Mater. Sci. Eng.A 483-484 (2008) 617-619.

[12] M.-W. Moon, K.-R. Lee, K.H. Oh, J.W. Hutchinson, Acta Mater. 52 (2004) 3151-3159.

[13] A. Strawbridge, H.E. Evans, Eng. Failure. Anal. 2 (1995) 85-103.

[14] M. Zhao, J. Zhou, F. Yang, T. Liu, T.-Y. Zhang, Eng. Fract. Mech. 74 (2007) 2334-2351.

[15] J. Wang, A.G. Evans, Acta Mater. 46 (1998) 4993-5005

[16] Y. Gachon, A.B. Vannes, G. Farges, M.C. Sainte Catherine, I. Caron, G. Inglebert, Wear 233-235 (1999) 263-274.

[17] S. Rossi, L. Fedrizzi, M. Leoni, P. Scardi, Y. Massiani, Thin Solid Films 350 (1999) 161-167.

[18] U. Wiklund, J. Gunnars, S. Hogmark, Wear 232 (1999) 262-269.

[19] J.S. Wang, A.G. Evans, Acta Mater. 47 (1999) 699-710.

[20] S. Lamri, C. Langlade, G. Kermouche, V. Martinez, Mater. Sci. Eng. A 527 (2010) 7912-7919.

[21] A.C. Sekkal, C. Langlade, A.B. Vannes, Mater. Sci. Eng. A 393 (2005) 140-146.

[22] S. lamri, C. Langlade, G. Kermouche, Adv. Mater. Res. 112 (2010) 73-82.

[23] W.C. Oliver, G.M. Pharr, J. Mater. Res. 19 (2004) 3-20.

[24] P.H. Mayrhofer, D. Music, T. Reeswinkel, H.-G. Fuß, J.M. Schneider, Acta Mater. 56 (2008) 2469-2475.

[25] J.A. Sue, A.J. Perry, J. Vetter, Surf. Coat. Technol. 68-69 (1994) 126-130.

[26] Abaqus Hibbitt, Version 6.5. (2004), USA.

[27] T. Belytschko, J.I. Lin, T. Chen-Shyh, Comput. Methods Appl. Mech. Eng. 42 (1984) 225-251

[28] F. Grange, G. Kermouche, C. Langlade, Ph. Gilles, Proceedings of the CFM2011 Conférence, September 2011, Besancon, France, AFM Ed., Courbevoie, France, $<$ http://hdl.handle.net/2042/46639 > .

[29] K.L. Johnson, Contact Mech., Cambridge University Press, 1985.

[30] P. Panjan, D. Kek Merl, F. Zupanic, M. Cekada, M. Panjan, Surf. Coat. Technol. 202 (2008) 2302-2305.

[31] P. Panjan, M. Cekada, M. Panjan, D. Kek-Merl, Vacuum 84 (2009) 209-214

[32] A. daS Rocha, T. Strohaecker, T. Hirsch, Surf. Coat. Technol. 165 (2003) $176-185$.

[33] M. Cekada, P. Panjan, D. Kek-Merl, M. Panjan, G. Kapun, Vacuum 82 (2007) 252-256. 